\title{
ANALISIS DE LOS PROCESOS DE INTERNACIONALIZACIÓN DE EMPRESAS COMPETITIVAS COLOMBIANAS
}

\author{
Analysis of the internationalization processes of Colombian competitive \\ companies/ Análise dos processos de internacionalização de empresas \\ competitivas colombianas
}

María Ximena Lemos Mejía y José Guadalupe Vargas Hernández ${ }^{2}$

\section{RESUMO}

Este trabalho contribui para o debate sobre a competitividade internacional na Colômbia, através de uma proposta que abranja a economia como um todo, mas que se concentra, especialmente, no sector manufactureiro colombiano (como gerador de valor agregado) e no ambiente económico no qual se encontra. Eles estão imersos, embora a competitividade que as empresas alcançam dependa, em grande parte, dos seus próprios esforços. Argumenta-se que os factores macroeconómicos, sectoriais e institucionais são um dos principais determinantes da produtividade alcançada pelas empresas, o valor agregado que geram e a enclave competitivo alcançado nos mercados nacionais e internacionais. Para este documento, informações estatísticas e documentais são usadas por agências governamentais e bibliográficas, a fim de contrastar e apoiar as questões acima referidas. Finalmente, pode dizer-se que os diferentes factores limitam a competitividade do sector manufactureiro colombiano.

Palavras-chave: Colômbia, Empresa, Produtividade, Competitividade, Política Comercial.

Recebido: Junho 2018

Aceitado: Julho 2018

\section{RESUMEN}

Este trabajo aporta al debate sobre competitividad internacional en Colombia a través de una propuesta que abarca a la economía en su conjunto, pero que especialmente se centra en las firmas del sector manufacturero colombiano (como generador de valor agregado) y el entorno económico en el que se encuentran inmersas. Si bien, la competitividad que logran las firmas depende en gran medida del esfuerzo propio de estas, se argumenta que los factores macroeconómicos, sectoriales e institucionales son uno de los principales determinantes de la productividad que alcanzan las firmas, del valor agregado que generan y del enclave competitivo que logran tanto en el mercado nacional, como en el

\footnotetext{
1 Centro Universitario de Ciencias Económico-Administrativas, Universidad de Guadalajara Maestría en Negocios y Estudios Económicos. Guadalajara, México. Correo electrónico: mximena.Im@gmail.com

${ }^{2}$ Centro Universitario de Ciencias Económico-Administrativas, Universidad de Guadalajara. Departamento de Administración. Guadalajara, México. Correo electrónico:
} jvargas2006@gmail.com 
Análisis de los procesos de internacionalización de empresas competitivas Colombiana.

internacional. Para el presente documento se hace uso de información estadística y documental de organismos gubernamentales y bibliográfica con el fin de contrastar y soportar lo expuesto. Se concluye finalmente que diferentes factores limitan la competitividad del sector manufacturero de Colombia.

Palabras clave: Colombia, empresa, productividad, competitividad, política comercial.

\begin{abstract}
This paper contributes to the debate on international competitiveness in Colombia through a proposal that covers the economy as a whole, but that especially focuses on the Colombian manufacturing sector (as a generator of added value) and the economic environment in which they are immersed. Although, the competitiveness that firms achieve depends to a large extent on their own efforts, it is argued that macroeconomic, sectoral and institutional factors are one of the main determinants of productivity reached by firms, the added value they generate and the competitive enclave achieved in both the national and international markets. For this document, statistical and documentary information is used from government agencies and bibliography in order to contrast and support the above. Finally, it is concluded that different factors limit the competitiveness of the Colombian manufacturing sector.
\end{abstract}

Key words: Colombia, company, productivity, competitiveness, trade policy

\title{
INTRODUCCIÓN
}

La acción no debe ser una reacción, sino una creación.

Mao Tse-Tung

Hoy por hoy, Colombia enfrenta la globalización con seria ausencia de competitividad. El rezago tecnológico que le caracteriza, el escaso desarrollo en infraestructura logística y del transporte, la poca innovación que llevan a cabo sus firmas en aspectos de producción y otros de tipo organizacionales, así como la baja participación del sector industrial y manufacturero en las exportaciones del país y en el PIB total han aportado al deficiente desempeño de Colombia en el escenario mundial (Consejo Privado de Competitividad-CPC, 2016).

La debilidad del sector productivo manufacturero y la caída constante, desde 1980 a 2017, de la relación Valor Agregado Industrial/PIB, la cual ha pasado de un $24 \%$ en los años 80 's, y antes de la apertura, a un 13\% para 2017 (Organisation for Economic Coperation and Development -OECD- 2017) reflejan 
la incapacidad de las firmas para producir bienes más valiosos y diferenciados en el mercado, determinando la falta de competitividad que hoy castiga a la realidad del comercio internacional colombiano y a la economía en su conjunto.

Esta casi inexistencia de sector industrial en Colombia, diversos autores la han denominado como un estadio de "desindustrialización prematura", reflejado por una creciente dependencia de la producción y exportación de hidrocarburos, esencialmente petróleo y carbón, forjados principalmente por el aumento en sus precios internacionales, pero que hace evidente que no se trate de una mejora en las capacidades y recursos de innovación y producción de las firmas colombianas (Penrose, 1959). Siguiendo lo anterior, el Departamento Nacional de Planeación y el Ministerio de Comercio Industria y Turismo, entes encargados de la política comercial del país, argumentan que hoy Colombia no está experimentando un proceso de desindustrialización, sino que se trata de una etapa de desaceleración económica, y que por tanto no se justifica la estridente preocupación y la exigencia de políticas industriales que piden a gritos académicos y empresarios para mejorar la competitividad.

El argumento en el que se basan estos organismos es que el Gobierno ya ha creado las bases macroeconómicas y los programas necesarios para que las empresas y la economía tengan un mejor desempeño. Aunque está demostrado que las variables macroeconómicas influyen de manera importante en el buen desempeño de una economía, estas no constituyen el único factor que la sustenta. Una mirada más detallada hacia las empresas y la productividad con que se desarrollan, es lo que realmente importa al momento de diseñar políticas que hagan ganar competitividad a una nación y no al revés (Porter, 1990; Krugman, 1990) pues esta hace que las capacidades de las firmas sean potenciadas. La sinergia y la estratégica mezcla que se genere entre las variables macroeconómicas y las que dependen exclusivamente de la firma, así como la simbiosis entre el Gobierno y las empresas, en función de la innovación y las nuevas ideas, son un importante camino para hacer a un país más próspero (McGrahan y Porter,1997). 
Análisis de los procesos de internacionalización de empresas competitivas Colombiana.

Por lo anterior, este ensayo procede de la siguiente manera: además de esta introducción, en la sección II se examinará el factor de competitividad asociado a la productividad promedio del trabajo en Colombia, así como también se hará una aproximación de esta variable en la industria manufacturera. En la sección III, se plantearán algunas condiciones macroeconómicas que impactan en la eficiencia y competitividad de las firmas; entendiendo a este último como el punto en donde se deben concretar las políticas y estrategias de desarrollo. En la sección IV se proponen algunos caminos o recomendaciones para un nuevo diseño de la política comercial que haga ganar competitividad internacional a las firmas colombianas. Se finaliza con una serie de conclusiones.

\section{PRODUCTIVIDAD DE LA SUMA TOTAL DE LOS FACTORES EN COLOMBIA}

\section{A. Índice de productividad del Trabajo en Colombia.}

El análisis de la Productividad Total de los Factores (PTF) como indicador de eficiencia viene desarrollándose desde los años 50's con los ensayos de Solow (1956), manteniéndose vigente con las ideas sobre la competitividad de Porter (1990), Banco Interamericano de Desarrollo (BID) (2010), Delgado, Ketels, Porter y Stern (2012) y Haltiwanger y Eslava (2017).

La premisa Productiva permite evaluar, en parte, el desempeño de las empresas y las industrias, puesto que son las primeras quienes conforman en consecuencia a las segundas (industrias) donde las firmas deben valerse de una serie de capacidades y recursos tangibles e intangibles internos que utilicen para aumentar la producción de sus bienes y/o servicios aunque sin desconocer la pertinencia que tiene sobre esto la existencia de ciertas variables exógenas (como las instituciones y las políticas) que determinan el uso eficiente de dichos recursos propios, (Vargas, Guerra y Jorquez, 2014; Peng and Heath, 1996; Peng, Pinkham y Chen, 2009) y que determinan otras competencias en la forma que le permitan ganar competitividad y mejorar su desempeño.

En este sentido, si miramos el desempeño de la productividad laboral agregada colombiana, tenemos que, tal como lo muestra la Figura 1, la improductividad laboral al interior de las firmas ha venido en aumento. Desde 2000 
Colombia ha mostrado una tendencia improductiva, donde una mayor cantidad de trabajadores ha sido necesaria para alcanzar el nivel de producción, y solo si se la compara con economías similares como México y Chile durante los años 20002006 muestra haber tenido un mejor desempeño productivo; sin embargo, después de 2006 esta ventaja se revierte y Colombia es superada por Chile y en 2011 por México, con diferencias importantes, sobre todo con este último, aunque no en extremo importantes desde 2014, donde parecen converger (Comisión Económica para América Latina y el Caribe, CEPAL, 2018). Aunque estos países vecinos muestran tener un comportamiento similar entre ellos, la situación al ser comparada con países avanzados como Suiza, Estados unidos o Dinamarca, hace que la brecha realmente exista y sea dramática.

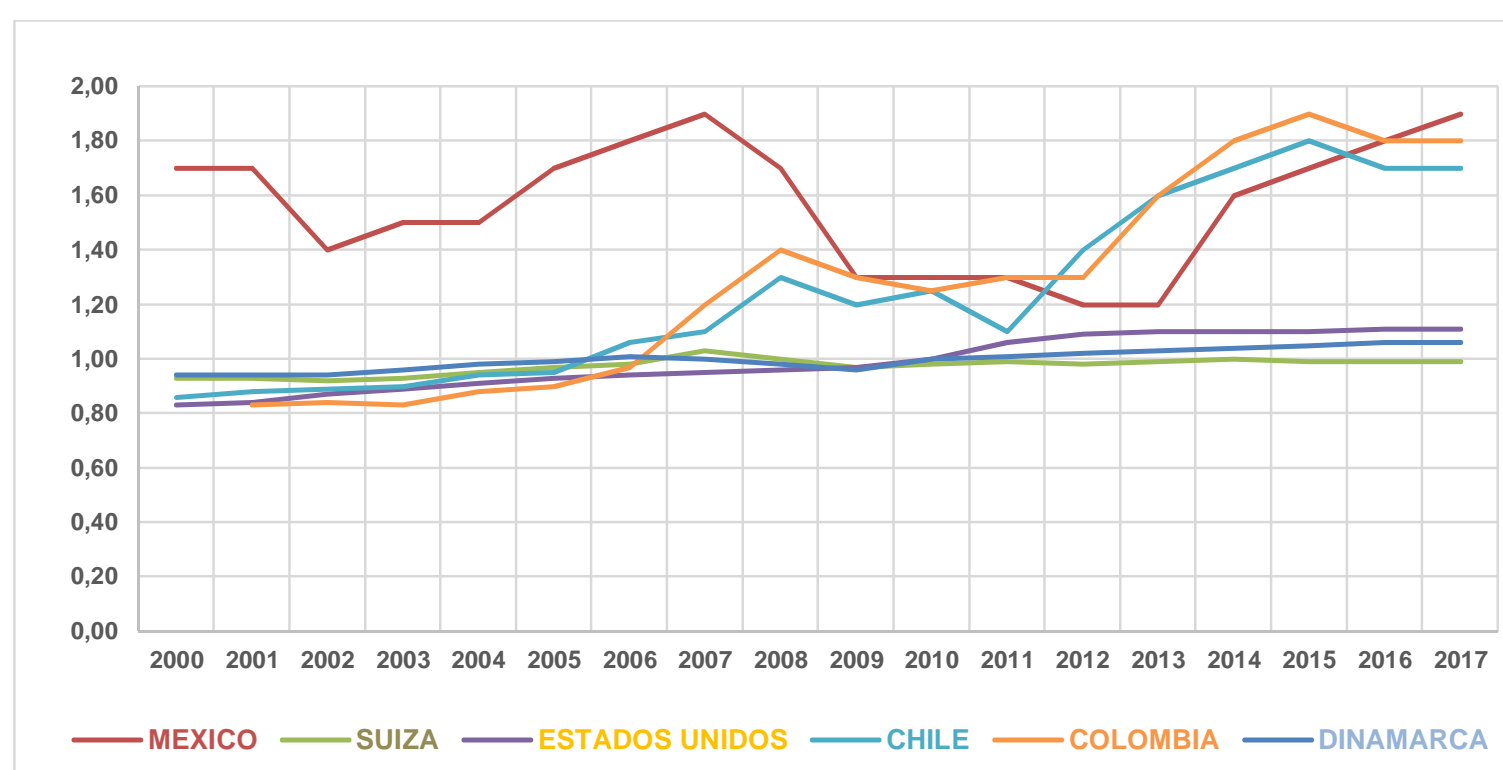

Figura 1: Productividad Laboral: Colombia, México, México, Estados Unidos, Suiza y Dinamarca. 2000- 2017. El índice está construido a partir de la producción nacional total (PIB en dólares constantes de 2010) y la cantidad de trabajo utilizada en esa producción.

Fuente: Organisation for Economic Co-operation and Development (OECD) (2017). Database: $\quad$ https://data.oecd.org/lprdty/labour-productivity-andutilisation.htm\#indicator-chart) Consultada en abril 10 de 2018.

De acuerdo a los datos anteriores, la productividad del trabajo en cada uno de los países ha ido en aumento, desde 2000 a 2017, con excepción del 2009 
Análisis de los procesos de internacionalización de empresas competitivas Colombiana.

cuando en todos los países cae este indicador como una consecuencia de la crisis de 2008, los países más avanzados presentan una tasa de crecimiento, de año a año, mayor que los demás países de la muestra. En Colombia, para los años 2010-2011, la productividad aumentó un 4,73\% y para 2011-2012 dicha productividad llegó al 4,96\%. Por el contrario, en países como Chile y México, en los períodos 2010-2011 dicha productividad varió en 7,11\% y 7,36\%, respectivamente y para los periodos 2011-2012, sus productividades se movieron en mayores proporciones, $10,85 \%$ Chile y $7,78 \%$ México. Lo que visto desde esta óptica supone un rezago importante de Colombia en la región en lo que respecta a la productividad del Trabajo.

Comparándo Colombia con países más avanzados como Suiza, Estados Unidos y Dinamarca, la brecha es también importante. Mientras nuestras tasas de productividad del trabajo varían de año a año en un 0,24\%, aproximadamente, ellos lo hacen en $1,50 \%$ y a un ritmo más acelerado; poniéndonos en seria desventaja para competir en los mercados internacionales. Detrás de este indicador de productividad, hay muchas variables que lo determinan. Por ejemplo, el sistema educativo del país y las normas que lo rigen, la flexibilidad laboral, la facilidad de acceso a la educación, la productividad de los sectores, entre otras. Por ejemplo, los costos de contratación y despido de las personas para las empresas son relativamente altos. Estudios de Fedesarrollo calculan que para un empleador los costes de contratación, diferente al sueldo, aumentan en un $73 \%$, debido a los aportes que deben hacer los patrones al Instituto Colombiano de Bienestar familiar (ICBF) y al Servicio Nacional de Aprendizaje (SENA).

Adicionalmente los trabajadores que se emplean por medio de un contrato deben obligatoriamente cotizar salud y pensión, lo que aumenta sus costos de transacción y esto se traduce para ellos en una disminución de sus ingresos y su consumo. Estas variables constituyen una razón importante para que las personas consideren contratarse y contratar en empresas no constituidas legalmente 0 informales.

Este es un tema crucial de competitividad si consideramos que a dichas empresas informales se les va a dificultar ser más productivas ya que el acceso al 
crédito, el cual permite aumentar la capacidad instalada, invertir en I+D, destinar presupuesto para el mercadeo y la publicidad, etc, será negado por los bancos puesto que estos sólo conceden créditos a empresas legalmente constituidas. También las empresas informales se verán con pocas oportunidades de mejorar ya que la atracción de personal capacitado se verá limitada debido a que si la empresa no es productiva esta no podrá pagar mejores salarios que persuadan a los trabajadores y por tanto en dichas empresas primará la mano de obra poco calificada y poco especializada, haciendo perder competitividad a las firmas al no contar con un acervo de capital humano que promueva Investigación, innovación y desarrollo.

Si se adicionan a la línea anterior el hecho que, y según estadísticas del Departamento Administrativo Nacional de Estadística (DANE)para finales de 2012 el $57 \%$ del sector empresarial se encontraba en la informalidad y el $51 \%$ de los trabajadores se encontraban empleados bajo estos esquemas de informalidad, tendríamos un problema más complejo y de carácter estructural, donde la calidad de nuestro capital humano se está viendo deteriorada al igual que nuestras empresas y donde las políticas hasta hoy llevadas a cabo no están yendo en la dirección correcta, pues se está generando un afecto adverso al logro de competitividad, promoviendo aún más la esfera improductiva de la informalidad.

\section{B. Índice de Productividad de la suma Total de los Factores de las industrias colombianas y en las regiones más representativas.}

Considerar el examen de las industrias más productivas en Colombia y su ubicación geográfica, es considerar la productividad misma de las firmas, y que implica un punto de partida para la creación estratégica de políticas comerciales, enfocadas en los sectores más prometedores para Colombia.

Según estudios del Banco de la República (2006) la productividad de la suma total de los factores productivos en Colombia difiere de industria a industria y de ciudad a ciudad. De acuerdo con la Figura 2 se tiene que para 2016 las industrias de bebidas y alimentos diversos, de papel, de materiales ferrosos y la 
de sustancias químicas y otros productos químicos (cosméticos), constituyen las industrias más productivas del país. Estas industrias se encuentran ubicadas principalmente en Bogotá, Cartagena, Cali, Medellín y Barranquilla y superan, en algunos casos, el índice promedio, de 100. Por otro lado, tenemos que las industrias menos eficientes o menos productivas son la industria del textil y de confecciones, la del calzado y la de fabricación de muebles y accesorios. En cada una de las regiones estudiadas estas industrias son el común denominador y se repite su patrón de ineficiencia.

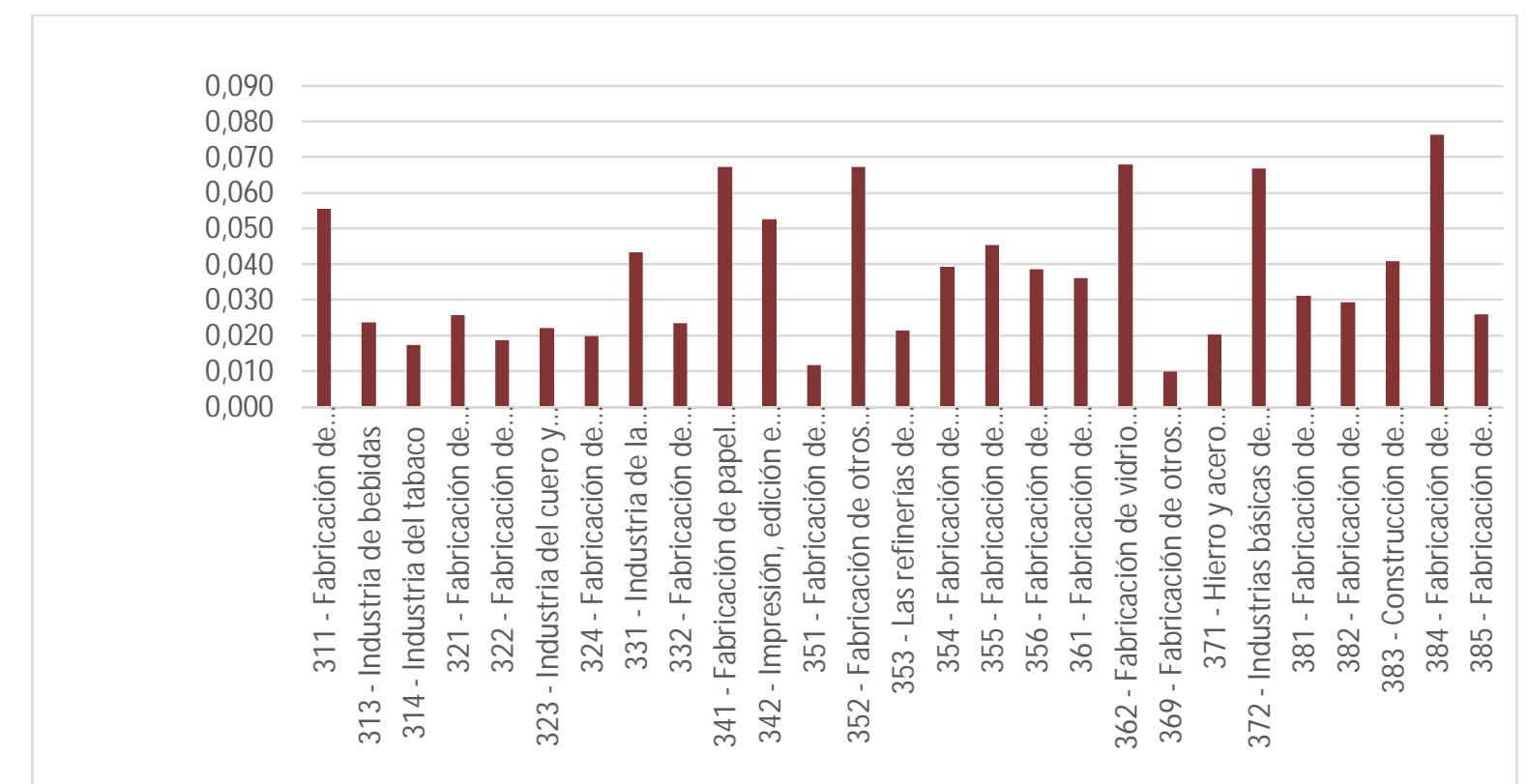

Figura 2: Índice de Productividad Total Factorial, por Industria. 2016. Elaboración propia con base en datos de CEPAL-Stat (Comisión Económica para América Latina y el Caribe, CEPAL 2018).

De igual forma se evidencian industrias que no presentan muy bajos indicies de productividad (son superiores a 50 ), como las de productos minerales no metálicos, fabricación de productos metálicos, construcción de maquinariaexcepto la eléctrica y la industria de material de transporte. Estas industrias, aunque no cuenten con elevados niveles de eficiencia, si son de tener en consideración, no sólo por mantenerse en el rango promedio de productividad, sino también porque son sectores que sirven de insumos para otras industrias y su 
especialización y desarrollo se convertiría en la especialización y el desarrollo de dichos sectores a los que provee (Vargas et-al, 2014). Así se logran aumentar los niveles de productividad agregada del país y con esto la competitividad para Colombia (Porter, 1981).

Este examen parcial de productividad puede dar lugar a plantear algunas reflexiones sobre las buenas o malas decisiones que se han tomado hoy en cuestiones de política comercial. Observando que existen industrias de baja productividad, como la de textiles, confecciones y calzado, no es lógico que hoy sean a estas mismas industrias a las que se protegen con aranceles, como se hizo en enero de 2013 y hasta hoy. Si bien es cierto que hay una competencia fuerte que es China y que esto afecta a los trabajadores de dichas industrias debido al desplazamiento que generan las importaciones y que crea una reducción en los puestos de trabajo internamente, también es cierto que desde la apertura esto se veía venir y que las políticas en pro de su aprovechamiento han sido de bajo impacto y que por el contrario han fomentado una mayor ineficiencia.

Ignorar el hecho de que unas industrias cumplan la función de proveer a otras, genera peores pérdidas que las previas al libre comercio. Siguiendo el caso de los textiles y el sector de confecciones, mientras a los textiles se los protege con un arancel ad-valorem del $5 \%$ al $10 \%$ por kilo importado, no sólo se está protegiendo una industria ineficiente, sino que también se están aumentando los costes para la fabricación de confecciones, industria que también se protege; haciendo que las tasas de protección para las confecciones sean inefectivas, continuando así el círculo vicioso de la improductividad, ya que ninguna de las dos industrias avanzarán, sobre todo la de confecciones, con tan altos costes de producción (Krugman y Obstfeld, 2010).

Por otra parte, tenemos que, mediante el Programa de Transformación Productiva, creado en 2004, la política también se ha enfocado en sectores eficientes como el de los químicos y sus preparaciones y el de material de transporte, haciendo que hoy las empresas de estas industrias, en mayor grado la de las preparaciones químicas, se hayan desarrollado bajo el principio fundamental de la innovación y la diferenciación, clave en el éxito de las firmas. En 
Análisis de los procesos de internacionalización de empresas competitivas Colombiana.

este punto las subsidiarias han jugado un papel fundamental, pues fueron estas las que se instalaron en Colombia con nuevas ideas de innovación es sus productos.

Aunque el Programa de Transformación Productiva (PTP) fue una aproximación para el desarrollo de industrias estratégicas en Colombia y con perfiles exportadores, se quedó corto en propuestas, ya que las necesidades más profundas de los sectores van más allá del posicionamiento que tengan dichos productos en el mercado internacional o de sus flujos internacionales; dichas necesidades son aún más trascendentales, buscando ser fuente de externalidades tecnologías positivas, sobre todo las de los motores que atañen a otras industrias, y que la articulación entre estas sea la oportunidad de generar más valor a la demanda y la oferta, nacional e internacional.

\section{EFICIENTES POLÍTICAS MACRO PARA LA EFICIENCIA MICRO}

Los ajustes estructurales son de impacto general con trascendencia en lo particular. Las fallas en aspectos generales clave de la competitividad en Colombia y que afectan el nivel micro, se dan en la baja actividad y poca solidez de las instituciones al frente de cada uno de los aspectos que repercuten en el país. Como consecuencia de tal inactividad se observan hoy deficiencias importantes en aspectos como la infraestructura carretera que conecta al país, donde, para 2017 , sólo el $40 \%$ del total de carreteras y calles se encontraban pavimentadas (World Economic Forum, 2018) y que por ende se traduce en un incremento en los costos de transacción de las firmas al momento de hacer negocios (Vargas et al, 2014).

Una política macroeconómica en cuanto al gasto público en I+D es de vital importancia. El Gobierno actual ha venido reduciendo el presupuesto que se daba a Colciencias, entidad que promueve la Investigación y el Desarrollo en Colombia. Hoy dicho Gasto en I+D es tan sólo un $0,53 \%$ del PIB, lo que representa un impedimento de gran envergadura para el desarrollo de la competitividad en el país. 
Si se compara este porcentaje con el de otros países de Sur-América, Brasil o Chile, quienes destinan aproximadamente un $1,2 \%$ de su PIB; o con países más avanzados como Estados Unidos, Alemania o Suecia, entre otros, quienes destinan del 3\%, en el caso de Estados unidos, al 5,5\%, en el caso de Alemania y Suecia, de su PIB (Banco Mundial, 2014). Se puede hallar las razones de porqué estos países si cuentan con productos y una canasta exportadora más diversificada y sofisticada que la nuestra; y que donde una moneda devaluada no constituye el factor primordial para ganar competitividad internacional.

Este último factor ha sido crucial en la pérdida de competitividad de los productos colombianos en el exterior, ya que la revaluación sostenida del peso en estos últimos años ha hecho que nuestros productos se encarezcan en el mercado internacional y se reduzcan nuestras ventas. Aunque una economía que se encuentre en proceso de desarrollo, como lo es la colombiana, una moneda devaluada es sinónimo de competitividad, es claro que ante la volatilidad con que se mueve el mundo esto es preciso cambiar, pues son este tipo de choques los que hoy nos sumergen en grandes problemas económicos y sociales.

El diminuto gasto colombiano en I+D no sólo lo hace el Gobierno, sino que también lo replican nuestras empresas, destinando tan sólo un $0,28 \%$, como proporción del PIB; empeorando aún más la situación colombiana. La inversión más prominente que hacen las firmas es en la modernización tecnológica, lo cual es importante pero no suficiente, pues hay otros factores también relevantes como la innovación y el desarrollo de nuevos productos y más sofisticados, lo cual se logra con investigación (Consejo Privado de Competitividad, 2016).

Así, se puede observar un mal enfoque en la estrategia tanto del Gobierno como de las empresas, ya que, y siguiendo las palabras de Porter (1996), la estrategia no es hacer lo necesario o algo igual y mejorarlo, o acudir a las últimas prácticas, como lo promueven las políticas actuales; sino que significa hallar un lugar donde nuestras industrias generen valor.

En este orden de ideas, si nos mantenemos enfocados en igualar procesos o hacer las cosas un tanto mejor no seremos capaces de brillar, el brillo no depende de si se es una empresa muy grande o muy pequeña, o una economía 
avanzada o emergente, el brillo está en función de la capacidad de mejorar y crear a la vez, generar diferenciales en los productos y servicios así como en la manera de gobernar, derivando valor para todas las partes pues de esto dependerá el éxito o fracaso de las empresa y como consecuencia de su nación.

Por todo lo anterior es evidente que una eficiente estructura macro, generada por las políticas de desarrollo que plantean los Gobiernos, son imperantes para un buen y productivo desempeño de las empresas nacionales. Los precios elevados del combustible, del cemento para el sector de la construcción, así como el bajo desarrollo en infraestructura eléctrica son también deficiencias que albergan nuestras políticas macroeconómicas. Estas problemáticas es fundamental solucionarlas ya que con una inadecuada infraestructura se aumentan los tiempos de transporte, se encarece el valor de los fletes y por ende aumenta el precio final de los bienes, lo que genera un juego de suma cero, donde los perdedores inevitables serán productores y consumidores, mientras las ganancias las obtendrá indiscutiblemente el sector del transporte y los proveedores de petróleo a costa de todos.

Es importante precisar que el objetivo no es beneficiar a los sectores más "importantes" o más productivos, sino crear valor en cada uno de los eslabones que componen a las cadenas productivas de las empresas, por tanto un enfoque hacia la industria de bienes no transables también es indispensable (Porter, 1990)

\section{PROPUESTA PARA EL DISEÑO DE UNA NUEVA POLÍTICA COMERCIAL QUE PROMUEVA LA COMPETITIVIDAD DE LAS FIRMAS COLOMBIANAS}

La competitividad no es algo estático, sino que radica en el mejoramiento e innovación constantes, y requiere que los programas para su consecución estén basados en estrategias que identifiquen puntos de flexibilidad que puedan ser modificables sin alterar su esencia, y que más bien creen ventajas comparativas dinámicas diferentes (Gunther, 2013).

La política comercial que ha caracterizado al país ha sido una política de reacción más que de creación. Su planteamiento ha sido en respuesta a los efectos que las nuevas condiciones internacionales han tenido al interior del país, 
sin poder tener el espacio para tomar decisiones más profundas o mejor enfocadas, que resulten en el discernimiento y la compresión de las necesidades de cada una de las industrias colombianas. Por tanto, para que la política comercial colombiana funcione, y con ella nuestra competitividad, esta no depende únicamente de un sólo ente gubernamental sino de muchos, ni depende de una sola política, depende de otras tantas necesidades como educación, salud, innovación, cultura, entre otras.

La competitividad no se separa del desarrollo social, es más, esta es una unión imperecedera que exige una sociedad saludable y en condiciones favorables en sus niveles de vida, para que así el aparato productivo también pueda crecer. Es por lo anterior que, se proponen algunos caminos para un mejor diseño de la política comercial y desarrollo en Colombia, que permitan ganar competitividad internacional:

1) La firma de Tratado de Libre Comercio (TLC) no garantiza que nuestros productos tengan acceso definitivo a los mercados internacionales. No considerar nuestras falencias y nuestras necesidades es el error más grande y que ha logrado opacar los beneficios de este tipo de acuerdos y por los que hoy la población declara su más plena inconformidad. Así como los gremios y productores de las industrias que compiten con las importaciones se agrupan y presionan las decisiones del Gobierno, es necesario que los exportadores cuenten con una igual concentración. La coordinación de estos grupos hace que sean mejor escuchadas las necesidades de sus miembros, ya que de manera dispersa sería más difícil lograrlo. La representatividad de estas nuevas agremiaciones es fundamental para que se hagan efectivas las peticiones y se trabaje eficientemente para ser incluidas en las políticas.

2) Profundizar la articulación entre Gobierno y empresa, es trascendental. Si las empresas necesitan de buenas vías para transportar suductos por qué no unir ambos esfuerzos para la construirlas apoyados a su vez en las bondades que ofrece el mercado de capitales. De esta forma ambos entes proporcionan beneficio general. La sinergia de las esferas Público-Privadas es fundamental si se 
Análisis de los procesos de internacionalización de empresas competitivas Colombiana.

quiere generar una transformación importante en la competitividad del país. Aquí los incentivos fiscales a las empresas por invertir en obras públicas se consideran como otra opción para estas ayudas mutuas.

3) Las empresas deben ser entendidas como el eje en donde se concreten las estrategias públicas. Es conveniente considerar que la función de Procolombia y de las Cámaras de Comercio del país deben orientarse a que las firmas y los inversionistas cuenten con estudios de mercado más específicos y sofisticados, y no tan generalizados como los de hoy. El modelo de las "cinco fuerzas" que estratégicamente planteó Michael Porter en los 90`s debe ser promovido enérgicamente en estas instituciones, ya que se podría acertar en proponer un mejor enfoque para la toma de decisiones. Con dicho conocimiento de cómo se relacionan las diferentes industrias y los mercados se puede llegar a entender hasta qué punto llegan los límites en las industrias y qué aspectos exige la rivalidad internacional a la industria y a sus empresas.

4) Cada vez las industrias tienes significados más amplios, tales definiciones tras el análisis antes descrito son necesarias, ya que se podrán ver con mayor detalle las tendencias y las necesidades actuales que precisan.

5) Al tener el anterior conocimiento detallado de las industrias, es preciso abrir paso a la creación de Clústeres y eficientarlos, donde la especialización de cada nivel que lo conforme sea vital. Dicho entendimiento también es determinante para la creación de la política industrial que necesita Colombia. Conocer la relación es clave para crear tasas de protección realmente efectivas y no repetir los errores que se encuentran en el PTP. La conformación de clústeres hace parte de dicha política industrial. Estas aglomeraciones permiten que se reduzcan costes de transporte, tiempos, aumente el flujo de insumos, de personas, debido a su cercanía geográfica, haciendo que el sector sea más productivo. El conocimiento de las industrias permite converger también según sus intereses. Dichos intereses pueden ser en función de los proveedores, del mercado meta, la tecnología utilizada, entre otros, lo que permite potenciar las capacidades de quienes conforman los clústeres y desarrollar su productividad. Esto hace que se puedan 
identificar espacios que las empresas aprovechen para diferenciarse de la competencia.

6) Una política que promueva y legitime el gasto del Gobierno en Investigación y el Desarrollo es para Ya! El Gobierno debe aumentar su gasto, por lo menos al 2,0\% del PIB en I+D. Aumentar el presupuesto que se da a Colciencias es clave, no se puede dejar dicho presupuesto a disponibilidad de recursos. Es un coste que se debe asumir obligatoriamente. Para aquellas empresas cuyas invenciones sean aplicables en otros sectores, la exención de impuestos puede darse por un periodo sostenido.

7) El ser un país abundante en Recursos Naturales ya nos hace ricos, pero el no saber qué hacer con ellos y dejarlos en su presentación más simple (como materias primas) es lo que nos estanca. Por esto la manufactura de este tipo de bienes debe ser el nuevo rumbo que tome este sector. Volverlo más productivo no depende, en principio, de los campesinos, depende también de las empresas más tecnificadas que puedan eficientemente producirlos y manufacturarlos en unión con los campesinos. Por esto, mejores condiciones en los préstamos, tasas de interés más bajas, desmonte del $4 \times 1000$, son algunas necesidades para financiar los proyectos que se dirijan a este sector y a la agregación de valor.

8) Manufacturar los bienes primarios es sólo el comienzo de la cadena. El siguiente paso es la producción de bienes de tecnología media para luego producir otros de tecnología alta. Es necesario ascender en estas cadenas generando valor agregado, Articularse en las cadenas globales de valor, debe ser un fin de la industrialización. Las empresas y multinacionales son las que están modificando hoy el comercio internacional y las formas de relacionarse unos países con otros. Por tanto, las iniciativas antes mencionadas van a permitir, en parte, a crear empresa y tener un aparato productivo mejor estructurado, para que una productiva Colombia internacional sea una realidad.

9) No se pueden negar los beneficios que genera la Inversión Extranjera en cualquier tipo de economía. Pero estos beneficios no podrán ser absorbidos si no se sabe a dónde dirigirla. Sectores hoy productivos como el de bebidas y productos alimenticios, así como el sector de los químicos, pueden ser grandes 
receptores de inversión extranjera directa (IED) con el fin de complementar sus técnicas. Requisitos de contenido local a dicha Inversión es válido en la medida en que las industrias locales que provean insumos sean especializadas y puedan beneficiarse de dicha IED. Para esto, especialistas en el tema, para este caso químicos, así como el diálogo entre ProColombia, entidades de la salud, y las empresas es imprescindible. Estos diálogos son precisos que sean desde ahora con el fin de que seamos nosotros los colombianos quienes decidamos que Inversión es la necesaria atraer y como obtener sus beneficios. La Inversión Extranjera guarda estrecha relación en la conformación de clústeres. Si, por ejemplo, para la IED que llega hoy al sector minero se organizaran clústeres que le proveyera herramientas, partes o piezas de sus máquinas, no se impondrían requisitos de contenido local, sino que la utilización de nuestros insumos sería algo natural. Para esto, una actitud y una aptitud de aprehender y crear conocimientos es fundamental para desarrollar estos esquemas productivos.

10) Si para nuestros exportadores no hay industria local que le provea insumos de alta calidad es necesario el desmonte de aranceles y exige un estudio profundo por parte de las entidades encargadas.

11) La productividad y la competitividad está en función de muchos más elementos y la educación es un factor que no se puede obviar en estos temasa, gracias a su trascendencia. Mejorar la calidad y la pedagogía en la educación primaria, secundaria y superior, y asegurar el acceso gratuito a estas, es vital para hacer de Colombia un epicentro de personas muy bien formadas, incluso desde edades tempranas, en distintas áreas del conocimiento y con habilidades técnicas de gran relevancia para el mercado laboral, con interesantes aportes a la sociedad.

12) El mercado laboral es también un indicador determinante de competitividad, por esto, políticas que forjen la flexibilidad laboral son importantes para eliminar trabas que incrementen la ineficiencia de este mercado. La reducción en los parafiscales a un total del $2 \%$ entre todos, sería un mejor incentivo para hacer que muchas empresas salgan de la informalidad y decidan contratar. 
13) Acceder al financiamiento internacional, por medio de la banca multilaterales internacional es necesario para hacer frente a las políticas de desarrollo que se requieren.

14) Hacer de nuestra demanda y nuestras instituciones apuestas de talla mundial es sin duda responsabilidad nacional. El no tener instituciones sólidas y exigentes con requerimientos que garanticen niveles de riesgo admisibles para los colombianos y extranjeros es un factor que no sólo hace competitiva a dichas instituciones, sino que también moldea nuestra demanda, haciéndola tan exigente como la internacional.

15) Los programas que se lleven a cabo y las valiosas ideas que se desarrollen, deben ser sin duda políticas de Estado, para así evitar el cambio constante en los objetivos que la discontinuidad genera tanto en las empresas como en los gobiernos. Se busca crear un sólo enfoque.

16) Finalmente, es necesaria una revolución en las ideas de quienes nos gobiernan, y aún más, de nosotros como sociedad. La creatividad está ahí para ser explotada e implementada en estas coyunturas que vive el país. Apelar a los viejos instrumentos de la política ya es evidente que no funcionan, los tiempos cambiaron, la igual que la magnitud de los obstáculos que estos conllevan, por tanto, las soluciones deben ser del mismo alcance. Se debe ser más atrevido en el diseño de políticas, jaunque aquí yo no lo represente!, se deben tocar conciencias y corazones donde se instalen "chips" más sofisticados y humanos, diferentes a los que ha implantado el pesimismo derivado de las pasadas guerras $y$ complicaciones que han limitado al país (aquí, la publicidad, la gestión del gobierno, cumplen una importante función), pues sólo así Colombia jugará y competirá como un Equipo de talla mundial.

\section{CONCLUSIONES}

La competitividad no es algo estático sino, algo dinámico; por tanto, la innovación y la creación deben ser una constante. 
Ser competitivos depende de una eficiente y flexible estructura de carácter macro y una de carácter micro. La primera está determinada por las políticas de desarrollo que se crean en el país, las cuales afectan a la esfera micro (empresas) y a la nación en su conjunto. Dichas políticas son de carácter económico, social, laboral, etc., las cuales construyen un marco que influye en la toma de decisiones de los distintos agentes de una nación. La segunda estructura la conforma el sector productivo, es decir las empresas. La efectiva mezcla que se da entre estos dos tipos de estructuras constituyen una razón de peso por la cual una economía puede ganar o perder competitividad.

El creciente auge que ha tenido el sector minero-energético en Colombia, forjado principalmente por el aumento en sus precios internacionales, ha hecho que se descuide la industria colombiana, puesto que ha generado una concentración de esfuerzos, políticas y ayudas hacia este sector, dejando desatendidas las necesidades de la esfera industrial.

La productividad de la suma total de los factores productivos en una nación (PTF) y el examen individual de la productividad del factor trabajo supone una medida de gran relevancia para evaluar la competitividad de un país. Colombia presenta altas brechas en la productividad del trabajo no sólo con los países desarrollados, sino con sus países vecinos. Mientras que en Colombia para 2012 la productividad del trabajo crecía en un $4,96 \%$, Chile y México crecían a tasas del $10 \%$ y del $7 \%$, respectivamente, lo cual genera un rezago importante para Colombia en la región, en términos de competitividad.

En un examen de la PTF en los sectores más importantes del país, se encuentra que sus productividades difieren de sector a sector y de ciudad a ciudad. Así los sectores más productivos son los de los alimentos procesados, papel y productos químicos, ubicados principalmente en las ciudades de Bogotá, Cartagena, Medellín, Cali y Barranquilla, lo que supone que debe hacerse una redistribución de los factores hacia dichos sectores potenciales. También contamos sectores improductivos, como el de las confecciones, los textiles y el calzado, a los cuales hoy se les protege, y que en palabras de Krugman y Obstfeld (2010), lo que hace esta protección es crear más ineficiencia y menor 
competitividad de la industria y de las empresas que en ella se desempeñan (Consejo Privado de Competitividad-CPC, 2016).

Las condiciones macro que ciñen al país son de verdadera ineficiencia. La inadecuada infraestructura, el bajo gasto público en $\mathrm{I}+\mathrm{D}$, el desequilibrio del mercado laboral, así como los costes de contratación y despido de trabajadores constituyen una barrera importante para el buen desempeño de las empresas. Concluyendo así que han sido las políticas las que han estado mal encaminadas y las que han permitido el pobre desempeño de las empresas y de la economía en su conjunto. Claro está no se le quitan culpas al sector privado por no crear estrategias de diferenciación de sus bienes y/o servicios.

\section{REFERENCIAS BIBLIOGRÁFICAS}

Banco de la República (2006). Productividad regional y sectorial en Colombia. Banco de la Republica. Colombia.

Banco Interamericano de Desarrollo-BID-. (2010). La era de la Productividad. Fondo de cultura Económica, México.

Banco Mundial (2014). El emprendimiento en América latina. Banco Mundial. Estados Unidos.

Comisión Económica para América Latina y el Caribe, CEPAL. (2018). Estadísticas Económicas. Cepal Stat. Santiago de Chile. Colombia Extraído de:http://estadisticas.cepal.org/cepalstat/WEB CEPALSTAT/estadisticasIndi cadores.asp?idioma $=$ e consulta: 05/06/2018.

Consejo Privado de Competitividad -CPC-. (2016) Productividad, la clave para el crecimiento colombiano. No. 3 Colombia. Extraído de: https://compite.com.co/informes/consulta: 05/06/2018.

Delgado, Mrcede; Ketels, Christian; Porter, Michael, y Stern, Scott. (2012). The determinants of national competitiveness. NBER Working Paper Series. Estados Unidos.

Gunther, Rita. (2013). The end of competitive Advantage: how to keep your strategy Moving as fast as your business. Harvard Business School Press. United States.

Haltiwanger, John y Eslava, Marcela. (2017). The Drivers of Life-cycle Growth of Manufacturing Plants. Meeting Papers (1540), Society for Economic Dynamics. United Kingdom.

Krugman, Paul. (1990). Competitiveness: A Dangerous Obsession. Foreign Affairs (24) 2. United States. (Pp:28-44).

Krugman, Paul y Obstfeld, Maurice (2010). Economía Internacional, teoría y política. 7a. Edición. Pearson. España.

McGrahan, Anita y Porter, Michel (1997). How much does industry matter, really? Strategic Management Journal, (18), United States. (Pp.15-30). 
Organisation for Economic Coperation and Development, OECD (2017). OECD Database: Economics Indicators. Washington. Extraido de: de:https://data.oecd.org//prdty/labour-productivity-andutilisation.htm\#indicator-chart consulta: 05/06/2018.

Peng, Mike and Heath, Peggy Sue (1996). The growth of the firm in planned economies in transition: Institutions, organizations, and strategic choices. Academy of Management Review, 21, United States. (Pp. 492-528).

Peng, Mike Li Sunny; Pinkham, Brian y Chen, Hao. (2009). The Institution-Based View as a Third Leg for a Strategy Tripod. Academy of Management Perspective, 23(4), United States. (Pp. 63-81).

Porter, Micheal E. (1996). What Is Strategy? Harvard Business Review, 74(6), United States. (Pp. 61-78).

Porter, Michael. (1990). The competitive advantage of nations. Harvard Business School Press. USA.

Porter, Michael. (1981). The contributions of industrial organization to strategic management. Academy of Management Review, (6), USA. (Pp. 609-620).

Penrose, Edith. (1959). A Theory of the Growth of the Firm. Wiley. USA.

Solow, Robert M. (1956). Technical Change and the Aggregate Production Function. The Review of Economics and Statistics, 39(3), USA. (Pp. 312320).

Vargas, José; Guerra, Ernesto; Bojórquez, Alberto y Bojórquez, Fernando. (2014). Gestión estratégica de las organizaciones. Insumisos Latinoamericanos, Argentina.

World Economic Forum. (2018). The Global Competitiveness Report 20172018. License: Creative Commons. Suiza. 